\title{
APROPIACIÓN SOCIAL DEL CONOCIMIENTO, INVESTIGACIÓN PARTICIPATIVA Y CONSTRUCCIÓN DE PAZ
}

\section{SOCIAL APPROPRIATION OF KNOWLEDGE, RESEARCH PARTICIPATORY AND CONSTRUCTION OF PEACE}

\author{
Mónica Lozano² \\ Mario Mendoza ${ }^{3}$ \\ Daniela Montaña ${ }^{4}$ \\ Rodrigo Parra Sandoval ${ }^{5}$ \\ Eureka Educativa \\ Universidad de East Anglia de Reino Unido \\ Universidad de Ibagué
}

\section{RESUMEN}

El presente artículo discute acerca de la capacidad que tiene la Apropiación Social del Conocimiento (ASC), de aportar desde la

escuela a la construcción de una paz sostenible en Colombia. A partir de la experiencia de trabajo con un grupo de maestros y maestras del sur del Tolima en Colombia se plantea cómo

1 Esta ponencia se basa en los resultados de la línea de educación del proyecto Escuela, Territorio y Posconflicto realizado en alianza entre la Universidad de Ibagué y Eureka Educativa en Colombia y la Universidad de East Anglia en el Reino Unido y financiado por el Ministerio de Ciencia y Tecnología en Colombia Contrato 227 de 2018 . El equipo de Eureka Educativa es el responsable de la línea de Educación del proyecto y está conformado por Mónica Lozano, Rodrigo Parra Sandoval, Daniela Montaña y Mario Mendoza.

$2 \quad$ https://orcid.org/0000-0002-9626-4152; https://scholar.google.com/citations?hl=en\&user=RhZWkv0AAAAJ; Correo: mlozano@eurekaeducativa.com

3 https://scholar.google.com/citations?user=SBPuMMUAAAAJ\&hl=es\&authuser=2 ; https://orcid.org/0000-0001-62018984 ; Correo: mmendoza@eurekaeducativa.com

4 https://scholar.google.es/citations?user=DexYHIQAAAAJ\&hl=es; https://orcid.org/0000-0001-8942-5950; Correo: dmontana@eurekaeducativa.com

$5 \quad$ Correo: rparra@eurekaeducativa.com 
el desarrollo de proyectos de investigación participativa sobre conflicto armado y paz, desde un enfoque orientado a lograr procesos de apropiación social del conocimiento, tiene la posibilidad de aportar a construir conocimientos y comprensiones individuales y colectivas acerca de la manera en que el conflicto armado se vivió en el territorio y cómo transformó a los maestros y a las comunidades. Se plantea que estas comprensiones son necesarias como un punto de partida para generar transformaciones sociales dirigidas a la construcción de una paz sostenible. El desarrollo de proyectos de este tipo constituye, en sí mismo, un proceso de intervención y de construcción de paz. Sostengo que laASC puede constituirse en una herramienta fundamental en el proceso de aportar a la construcción de una paz sostenible, en especial en contextos que, como el colombiano, han vivido el conflicto armado. La ASC puede contribuir a la necesaria reflexión académica sobre el conflicto y la paz de diferentes maneras. De un lado, agrega miradas novedosas, construidas desde los mismos actores locales. De otro lado, promueve la generación de estrategias, dirigidas a la construcción de una paz sostenible que responda a las dinámicas y contextos en los que se desarrollan los procesos de intervención social.

PALABRAS CLAVE: Apropiación social del conocimiento, construcción de paz, investigación participativa en educación, posconflicto, escuela, territorio, educación para la paz.

A partir de la década de los 90 del siglo pasado en América Latina se inició el uso del término de Apropiación Social del Conocimiento (ASC) y Apropiación Social de la Ciencia y la Tecnología (ASCT), para designar una manera especial de intervenir en las relaciones entre conocimiento científico y ciudadanía en un contexto que abogaba por los procesos de democratización del conocimiento científico y tecnológico en nuestros países.
Si bien en ese momento, había un consenso generalizado de la necesidad de propender por unas nuevas formas de entender las relaciones de ciencia y sociedad y una crítica desde algunos sectores a los modelos deficitarios que habían guiado la relación entre los científicos y sus públicos, la discusión acerca de qué es ASC, cuáles son sus métodos, cómo se concibe a los públicos y cuál es la relación entre ciencia y sociedad que se propende desde este acercamiento, está lejos de estar cerrada (Lozano, 2005; Daza y Arboleda 2007; Franco-Avellaneda, M. y Perez-Bustos, T. 2010; Hermelin, 2011; Maldonado, 2011; LozanoBorda y Pérez-Bustos, 2012, Daza, 2013).

Existen, sin embargo, lo que a mi juicio podrían señalarse como acuerdos acerca de algunas características de la ASC, en medio de lo que podríamos denominar un campo en ciernes:

1) La importancia de que la investigación científica realizada desde las universidades y otros ámbitos del mundo académico responda a problemas que son reconocidos y enunciados como tales no solo por la comunidad científica, sino también por los actores sociales del contexto en el que se desarrolla la investigación.

2) El reconocimiento de que además del conocimiento científico existen otro tipo de conocimientos y experticias y que, en los procesos de ASC, estos conocimientos y saberes resultan tan importantes como los desarrollados desde el ámbito académico.

3) El reconocimiento de que la solución de problemas sociales implica el uso de conocimientos, pero debe tener en cuenta al mismo tiempo, sistemas de valores y creencias de las comunidades y organizaciones sociales que intervienen y participan en los proyectos.

4) La inclusión de modelos democráticos de comunicación pública de la ciencia que fomenten la participación pública en la ciencia en 
los distintos momentos del proceso investigativo y no solo al final como estrategia de difusión de resultados.

Para el proyecto partimos de definir la ASC como el proceso a través del cual el conocimiento, científico y de otro tipo, es apropiado por personas y grupos sociales para la comprensión del entorno y la transformación de problemas sociales desde una perspectiva local, situada y de desarrollo humano sostenible 6 . Los procesos de ASC implican la generación colectiva de nuevos conocimientos y un ejercicio de participación ciudadana en la solución de problemas que les atañen.

Por su parte, el concepto de paz se ha ido transformando a partir del desarrollo de los estudios sobre paz, pero también como resultado de la agenda internacional impulsada por la Organización de Naciones Unidas (ONU) sobre el tema ${ }^{7}$. La paz sostenible se reconoce como la búsqueda de paz articulada con los esfuerzos por alcanzar los Objetivos de Desarrollo del Milenio (ODM). A diferencia de conceptos anteriores en los que la paz aparece vinculada a la guerra y es definida desde ella, en este nuevo concepto se encuentra relacionada con la necesidad de generar enfoques preventivos -y aquí la importancia de la educación para la paz-, el desarrollo de procesos democráticos y participativos en su consecución y la búsqueda de un desarrollo humano sostenible: la búsqueda de la paz involucra la reflexión sobre las futuras generaciones.

Gran parte de las iniciativas dirigidas a la construcción de paz desde la escuela y que se desarrollan en los contextos de posconflicto, parten de modelos construidos fuera de los

$6 \quad$ Este último entendido desde la formulación de A. Sen (2000) cómo la posibilidad de desplegar las capacidades, la posibilidad real de ser y hacer aquello que considera valioso sin que implique comprometer las posibilidades de las generaciones futuras.

$7 \quad$ Para una revisión de la manera en que ha evolucionado el concepto de paz en Naciones Unidas ver Gómez (2011). territorios que buscan ser implementados con la participación de los maestros y las escuelas, como ejecutores de las propuestas (Buckland, 2005; Novelli y Lopes Cardozo, 2008; Hawrylenko, 2010). Este tipo de esquemas quita a los maestros la posibilidad de incidir de manera autónoma en el proceso de reconstrucción social y genera lo que podríamos entender como un doble desposeimiento: aquel generado durante el conflicto armado y otro, el generado durante el posconflicto. El problema fundamental es que, con frecuencia, los programas se desarrollan en contextos que poco o nada tienen que ver con la situación de las escuelas y los maestros y parten de una visión del territorio, del conflicto y la paz entendidos como conceptos unívocos (la que asume el programa y que responde al marco teórico adoptado).

En esta presentación quisiera detenerme en la relación que existe entre ASC y construcción de paz y las opciones que este enfoque traería en el contexto actual del posconflicto en Colombia. Sostengo que la ASC puede constituirse en un proceso que puede aportar a la construcción de una paz sostenible, en especial en contextos que, como el colombiano, han vivido el conflicto armado. La ASC puede contribuir a la necesaria reflexión académica sobre el conflicto y la paz de diferentes maneras. De un lado, agrega miradas novedosas, construidas desde los mismos actores locales. De otro lado, promueve la generación de estrategias, dirigidas a la construcción de una paz sostenible que responda a las dinámicas y contextos en los que se desarrollan los procesos de intervención social.

Esto no es solamente útil para los actores locales que participan en la investigación, sino que, además considero que este es quizá su elemento más importante, aporta a procesos de transformación de quienes impulsan el desarrollo de las iniciativas en el territorio, al apelar a la orientación simétrica (de doble vía) 
de los procesos de ASC. Como una manera de introducir la reflexión, quisiera referirme al Proyecto Escuela, Territorio y Posconflicto que desarrollamos de manera conjunta en una alianza que involucra a la organización Eureka Educativa y a la Universidad de Ibagué en Colombia y a la Universidad de East Anglia, en el Reino Unido, y que se desarrolla en el sur del Tolima, en los municipios de Ataco, Chaparral, Planadas y Rioblanco.

\section{EL PROYECTO}

Escuela, Territorio y Posconflicto se formula en el marco del proceso del posconflicto $y$ busca aportar a la construcción de una paz sostenible por medio de un esquema de trabajo que involucra la realización de procesos de investigación de tipo participativo con maestros y miembros de organizaciones comunitarias, a partir del diseño de propuestas para la construcción de paz basados en los resultados de la investigación y la implementación de estas propuestas (ver esquema).

El proyecto se desarrolla en el sur del departamento de Tolima, Colombia, en los municipios de Ataco, Chaparral, Planadas y Rioblanco e involucra escuelas y organizaciones sociales del sector rural y urbano de los municipios.

El sur del Tolima es un lugar muy especial en la historia del conflicto armado colombiano, no solo aquí se produce el surgimiento de las Fuerzas Armadas Revolucionarias de Colombia, FARC, sino que durante los más de 60 años que duró el conflicto armado, el grupo guerrillero, al igual que otros actores armados, tuvieron presencia en la zona. La ubicación del departamento y en especial de esta zona, la convirtió en un enclave importante y en un corredor que permitía a las FARC su tránsito hacia distintos lugares del país: la costa pacífica, los departamentos de Cauca, Valle, Huila y hacia el centro del país: Cundinamarca y Meta. Durante muchos años, la región fue considerada una zona guerrillera, lo que generó estigmatización y también abandono estatal. Actualmente es reconocida como una de las zonas priorizada con intervenciones sociales en el marco del proceso de paz.

En la línea de educación se han vinculado 39 maestros y maestras pertenecientes a instituciones educativas (IE) urbanas y rurales de cada uno de los cuatro municipios: en Chaparral la IE Manuel Murillo Toro (zona urbana) y IE La Risalda (zona rural) en la vereda del mismo nombre; en Ataco la IE Martín Pomala (urbana) y la IE Santiago Pérez en la vereda del mismo nombre (rural); en Rioblanco, la IE Francisco Julián Olaya (zona urbana) y la IE San Rafael, ubicada en el corregimiento de Herrera, zona rural del municipio; y en Planadas la IE Santo Domingo Savio (urbano), en este último municipio el sector rural es asumido por la Sede EI Silencio de la misma IE. De manera conjunta con los maestros se responde a las siguientes preguntas: ¿qué le hizo el conflicto y en especial el armado, a la escuela, a los maestros y a la comunidad educativa? ¿Cómo 60 años de conflicto armado han incidido y transformado la capacidad de construcción de paz de la escuela y sus maestros?

Para responder a la pregunta de investigación se utilizó un método cualitativo - interpretativo que involucró a los maestros como coinvestigadores en un proyecto que tenía definidos al inicio la pregunta y la metodología del proyecto. Los maestros y maestras fueron invitados a participar a través de la elaboración de narrativas biográficas individuales sobre la manera en que vivieron durante su vida el conflicto armado. Estas narrativas, aunque individuales, fueron elaboradas en talleres colectivos -encuentros en cada uno de los municipios que reunián a los maestros investigadores de las escuelas urbanas y rurales de ese lugar- $y$ en donde los maestros compartían no solo el resultado sino también el proceso de elaboración. 
Además de estos encuentros (talleres in situ) se tenían también talleres generales en los que los maestros y organizaciones de todos los municipios se reunían para compartir los avances de los procesos de cada grupo.

En la línea educativa, durante el desarrollo del proyecto se definieron tres grandes componentes, cada uno con instrumentos específicos para la recolección de información. El primero relacionado con el currículo y la manera en que incorpora la educación para la paz. El segundo que indaga sobre la manera en que la comunidad educativa se ha afectado con el conflicto: interroga la visión de paz y conflicto que tienen los maestros y los niños, su visión de territorio, de escuela, futuro y la manera en que se transforman en el tiempo a partir del conflicto. Para el desarrollo de este componente se utilizaron las narrativas biográficas: la escritura biográfica, la realización de cartografías corporales, la producción de narrativas digitales, entre otros. El tercer componente, en el que se enfoca este artículo, se estructura con una meta reflexión acerca del proceso vivido y la capacidad que tiene el desarrollo de procesos de investigación colaborativa, para generar procesos de apropiación social del conocimiento y generar transformaciones que apunten a la construcción de una paz sostenible.

Durante los talleres in situ con maestros, que eran diseñados y dirigidos por el equipo coordinador de la línea educativa, se realizaba un ejercicio de análisis e interpretación colectiva de las producciones y estos resultados parciales eran incorporados en los talleres con los otros equipos. En los talleres generales, el equipo coordinador "devolvía" usando como estrategia la exposición museográfica los resultados y análisis realizados en cada uno de los municipios, lo que motivaba relecturas involucrando ya no solo a los maestros sino también a todos los participantes del proyecto general (organizaciones y equipo coordinador de la línea comunitaria).

Al finalizar, el equipo coordinador realizó el ejercicio de análisis global de la información usando las categorías principales del proyecto: identidad, territorio, conflicto y paz, teniendo como eje transversal la transformación. Los resultados e interpretaciones fueron devueltas a los maestros a través de talleres y ejercicios de lectura compartida y ajustados hasta tener los resultados finales ${ }^{8}$.

\section{SOBRE APROPIACIÓN SOCIAL DEL CONOCIMIENTO Y CONSTRUCCIÓN DE PAZ EN LA ESCUELA: LOS APRENDIZAJES DEL PROYECTO}

Los resultados del proyecto Escuela, Territorio y Posconflicto en la línea educativa están próximos a su publicación ${ }^{9}$. Quisiera, sin embargo, detenerme en algunos de los aprendizajes importantes que arroja esta experiencia para la reflexión acerca de la potencialidad que tiene una aproximación desde la investigación participativa como una herramienta para la apropiación social del conocimiento y su capacidad para aportar a la construcción de paz en el ámbito educativo $y$, en general, en otros ámbitos que se apoyan en la investigación social sobre la paz y el conflicto.

\section{LA INVESTIGACIÓN PARTICIPATIVA Y LA APROPIACIÓN SOCIAL DEL CONOCIMIENTO}

Quizá el punto de partida es establecer la relación que existe entre la investigación participativa que utilizamos en el proyecto y los procesos de apropiación social del conocimiento. En el proyecto, la Investigación Participativa (IP) la asumimos como un medio a partir del cual se intenta alcanzar el propósito que persigue la ASC, es decir, que los actores sociales hagan suyo el conocimiento (científico o de distinto tipo) para comprender y transformar su mundo

$8 \quad$ Este es el proceso que se está desarrollando en el primer semestre de 2021.

9 http://eurekaeducativa.com/etp 
en direcciones que valoran y deciden libremente.

En el proyecto, la investigación se plantea desde un enfoque colaborativo que permite que los maestros con el acompañamiento de los investigadores externos, puedan reflexionar acerca de la manera en que el conflicto se desarrolló en el territorio y la incidencia que ha tenido en las escuelas y en la vida de los niños, pero además identifica la manera en que los maestros, durante todo el proceso, han sido agentes de cambio y transformación en el territorio.

El uso de la investigación participativa (o colaborativa en el sentido en que es asumida en el proyecto) ha sido utilizada con frecuencia en el desarrollo de proyectos de ASC, uno de los ejemplos más importantes es el Programa Ondas del Ministerio de Ciencia y Tecnología en Colombia, en el que la investigación realizada por los niños, niñas y jóvenes con el acompañamiento de sus maestros y acompañantes externos, se convirtió en una herramienta dirigida a lograr la apropiación social del conocimiento y el desarrollo de una cultura de ciencia, tecnología e innovación en la población infantil y juvenil del país.

Un enfoque desde la ASC facilita la construcción de abordajes conceptuales y metodológicos propios sobre construcción de paz

Desde mediados del siglo pasado los estudios sobre paz y conflicto, a la vez que organismos internacionales han desarrollado una serie de aproximaciones conceptuales, son usados con mucha frecuencia a la hora de generar propuestas dirigidas a construir paz desde la escuela y se ubican en el amplio registro de los proyectos de educación para la paz. En el proyecto ETP, el uso de la investigación participativa de tipo colaborativo con los maestros como investigadores, nos planteó la posibilidad de abordar las comprensiones del conflicto y de la paz que han construido los maestros y la escuela en su trabajo cotidiano, lo que aporta a la construcción de marcos explicativos útiles, no solo al momento de comprender la complejidad de un conflicto a gran escala y la manera en que afecta a la escuela, sino también los retos de construir paz en el marco del post acuerdo.

Durante el desarrollo del proyecto emergen conceptos de paz que están relacionados con las historias personales, la escuela y la manera en que interpreta su mandato de formación para la paz y la estrecha relación entre paz y conflicto armado en el territorio. Muchos de estos conceptos son similares a los que se han trabajado en la literatura sobre el tema: la paz como ausencia de conflicto o la paz como un estado mental individual o la paz como búsqueda de desarrollo personal, de formación en valores y construcción de herramientas para la vida que permiten resolver los conflictos sin violencia. Sin embargo, también emergen otros sentidos: visiones de la paz como inacción, como el silencio en un mundo lleno de gritos, como la habilidad de permanecer callados y evitar "meterse en problemas", o la paz como acción, como capacidad de transformación que asumen los actores sociales (en este caso los maestros), entre otros significados.

De la misma manera el proyecto permite evidenciar las relaciones que se establecen entre los distintos tipos de violencia a la que están expuestos los maestros y las escuelas en el territorio. Desde las violencias 'más visibles' y que en general son documentadas en la literatura sobre el tema, como lo son las violencias directas ejercidas por los actores armados contra la escuela y los maestros, que podemos representar en amenazas, intimidaciones, la exposición a la ocupación de las escuelas o el quedar en medio de los bombardeos o fuegos cruzados. Hasta otras formas de violencia que difícilmente se reconocen como tales en los programas de educación o construcción de paz y que llegan a la escuela con el posconflicto. Aquí 
hago referencia, por ejemplo, a la vulnerabilidad a la que están expuestas las escuelas y los maestros en los sectores rurales más apartados y que generalmente han vivido el conflicto con mayor fuerza: maestros viviendo en medio de una inestabilidad laboral e institucional que permanece a pesar de que los enfrentamientos han terminado. Violencias étnicas, de género, sexuales que permanecen y que constituyen formas complejas y que a veces, el énfasis en el conflicto armado invisibiliza o incluso normaliza.

El uso del enfoque que prioriza la ASC, el diálogo de saberes, el desarrollo de procesos participativos y la orientación a la acción, permite en estos contextos de conflicto, aportar a la construcción de marcos conceptuales e interpretativos sobre la paz y el conflicto, que pueden ser más pertinentes y adecuados localmente, y que son reconocidos como pertinentes por los actores sociales que viven $y$ permanecen en el territorio.

Un enfoque desde la ASC permite la participación de maestros, estudiantes y la escuela en general en procesos de investigación sobre su mundo y en la construcción de visiones compartidas sobre una paz sostenible

Uno de los elementos centrales que caracteriza los proyectos de ASC es la importancia central que se le asigna a la participación de los actores sociales en las acciones y decisiones que les competen y cuyos resultados les afectan de manera directa. Una de las situaciones comunes en el desarrollo de los proyectos es la falta de participación de los maestros en procesos que son muy importantes para ellos. Weldon (2010) al referir la experiencia en Ruanda y Sudáfrica muestra cómo las iniciativas gubernamentales en general desconocen el papel de los maestros y los ve como personas que desarrollan propuestas.

Un elemento fundamental del ejercicio de ASC que se realiza dentro del proyecto es promover la participación de los actores diversos (maestros, niños, padres, pero también miembros de organizaciones sociales y líderes comunitarios) en la búsqueda de comprender el conflicto (¿qué nos pasó? ¿por qué? ¿cómo nos afectó y sigue afectando? ¿qué aprendimos?), sino también -y esto es muy importante-, en la posibilidad de intervenir sobre estos problemas usando las fortalezas construidas durante el proceso.

En ocasiones las intervenciones van desde situaciones puntuales, casi que invisibles para alguien externo, porque se suceden dentro de cada uno: la posibilidad de escuchar de manera compasiva la visión del otro, de descubrir la "humanidad" que está en personas que hemos visto con recelo, el reconocer y reconciliarse con la propia historia. Otras intervenciones se producen en el entorno cercano, la familia, los amigos, la práctica pedagógica cotidiana en la escuela. Finalmente se evidencian hasta transformaciones sociales, relacionadas con el desarrollo de propuestas dirigidas de manera específica a construir paz interviniendo política, pedagógica y socialmente sobre el entorno.

Involucrar a los maestros como investigadores y no solo como "sujetos" de la investigación, se justifica desde los siguientes argumentos:

- Los maestros poseen un amplio conocimiento sobre el mundo escolar, sobre los intereses, valores y condiciones en las que se desarrolla el acto educativo. Su visión como coinvestigadores permite enriquecer la investigación tradicional al introducir nuevas preguntas y perspectivas que en general desaparecen cuando se hace una investigación "externa".

- $\quad$ El conocimiento especial que tienen los maestros del territorio, la escuela, las relaciones comunitarias, permite una mejor evaluación del potencial de oportunidades, opciones y riesgos de alternativas de solución a los problemas identificados, aportando a una mayor capacidad 
de sostenibilidad de las iniciativas en el tiempo.

- El desarrollo de procesos de investigación realizados con los maestros, no solo contribuye al posicionamiento social de su labor en la institución educativa y en la comunidad, sino también contribuye a los procesos de aceptación y legitimación de los hallazgos.

Un enfoque de ASC permite la construcción de agencia

El desarrollo de procesos de ASC permite que personas y comunidades que vivieron durante tanto tiempoel conflicto puedan sercomprendidos más allá de una visión de víctimas del conflicto armado o del abandono estatal o las violencias institucionales. Permite una comprensión, no tanto para las personas del territorio, sino para la academia y los organismos que desarrollan propuestas de construcción de paz, de que nos encontramos con agentes, personas que han vivido el conflicto y que durante todo este tiempo desarrollaron estrategias que les han permitido construir posibilidades de paz aun en medio del fuego cruzado. En el caso de los maestros esto es claro: muchos de ellos, junto con las comunidades, allí donde no había, han construido escuelas; han convertido las experiencias de las violencias de diverso tipo, en posibilidades de generar propuestas para evitar la perpetuación de la guerra: la maestra que vivió violencia infantil cuando niña y que luego pasa a ser madre maltratadora, se convierte en alguien que, a partir de descubrir los patrones que se perpetúan, puede ella misma construir, desde su espacio familiar, desde el aula y luego desde el municipio, propuestas dirigidas a luchar contra el conflicto armado. La maestra que fue víctima de la violencia de género, puede darse cuenta de que esta es una forma naturalizada en que se han asumido las relaciones de pareja y que, además, se repite en las distintas generaciones en su familia, y logra, a partir de ese "darse cuenta" transformar su vida, pero también las de sus alumnos y de la comunidad.

\section{A MANERA DE CONCLUSIÓN}

El desarrollo del proyecto nos muestra caminos a través de los cuales se pueden generar propuestas dirigidas a aportar a la construcción de una paz sostenible basadas en la generación, uso y apropiación social del conocimiento sobre el conflicto y la paz en territorios que han vivido la guerra. El uso de la investigación participativa como un medio para la apropiación social del conocimiento permite: a) generar abordajes conceptuales y metodológicos para investigar e intervenir sobre paz, que parten de un contexto social y situado; b) facilitar la participación de distintos actores sociales que han vivido el territorio y que en el marco del pos acuerdo permanecen allí, esta participación constituye un elemento fundamental en el proceso de pensar la sostenibilidad de las estrategias; 3) el enfoque de la investigación participativa y de la búsqueda intencional de la apropiación social del conocimiento, aporta al desarrollo de la agencia de los actores sociales.

\section{REFERENCIAS}

Buckland, P. (2005), Reshaping the Future, Education and Postconflict Reconstruction, Washington: The World Bank.

Daza, S. y T. Arboleda (2007), Comunicación Pública de la Ciencia en Colombia: ¿Políticas para la democratización del conocimiento?, Signo y Pensamiento, 25, pp. 101-125.

Daza, S. (2013). La Apropiación Social de la Ciencia y la Tecnología como un objeto de frontera, en Vogt C, Pallone S, Barata G y Kanashiro M (org.), Comunicação, Divulgação e Percepção de Ciência e Tecnologia (C\&T), Petrópolis, RJ: De Petrus et Alii; Brasília, DF: CAPES. 
Franco-Avellaneda, M. y Pérez-Bustos, T. (2010), Tensiones y convergencias en torno a la Apropiación Social de la Ciencia y la Tecnología en Colombia, en Pérez-Bustos, T y M. Tafur Sequera (eds.), Deslocalizando la Apropiación Social de la Ciencia y la Tecnología: Aportes desde Prácticas Diversas, Bogotá, MalokaColciencias, pp. 9-23.

Gomez, C. (2017), Paz sostenible: nuevo paradigma para el trabajo de la ONU, Revista Mexicana de Política Exterior, 110, pp. 61-77.

Hermelin, D. (2011), Un contexto para la comunicación pública de la ciencia y la tecnología en Colombia: de las herencias eurocéntricas a los modelos para la acción, Co-herencia, 8, (14), pp. 231-260.

Hawrylenko, J. (2010), Education in Postconflict Societies (Thesis of master degree). Athabasca University, Athabasca, Alberta.

Lozano, M. (2005), Programas y Experiencias en popularización de la ciencia y la tecnología. Panorámica desde los países del Convenio Andrés Bello, Bogotá, Convenio Andrés Bello.

Lozano-Borda, M. y Pérez-Bustos, T. (2012). La apropiación social de la ciencia y la tecnología en la literatura iberoamericana. Una revisión entre 2000 y 2010 . Redes, 18, (35), pp. 45-74.

Maldonado, O. (2011), "Conocimiento y política de lo público. Una contribución a la definición de Apropiación Social del Conocimiento desde el campo de la política pública", en Pérez-Bustos, T. y M. Lozano Borda (eds.), Ciencia, Tecnología y Democracia: Reflexiones en torno a la Apropiación Social del Conocimiento, Medellín, Universidad EAFIT-Colciencias, pp. 147-156.

Novelli, M. y Lopes Cardozo M. (2008), Conflict, education and the global south: New critical directions, International Journal of Educational development, 28, (4), pp. 473-488.
Sen, A. (2000), Desarrollo y Libertad, Barcelona: Planeta.

Weldon, G. (2010), Post conflict teacher development: Facing the past in South Africa, Journal of Moral Education, 39, (3), pp. 353-364. 